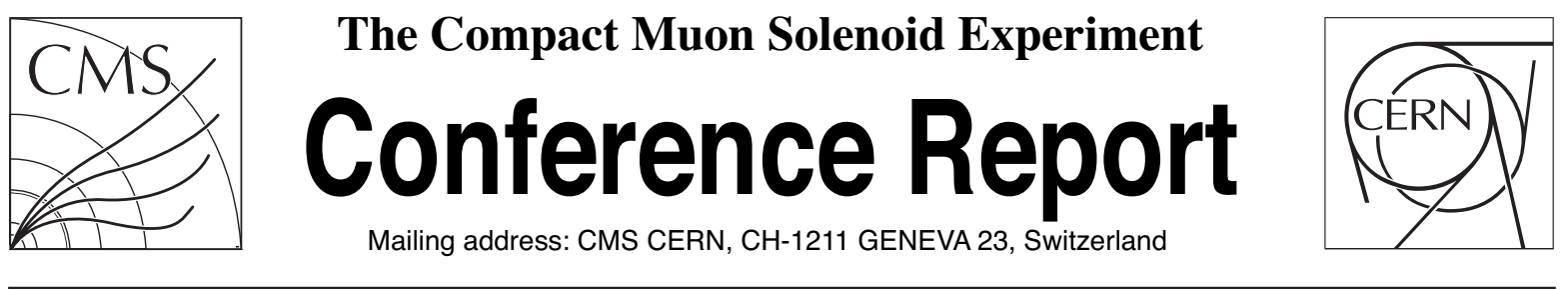

08 November 2013 (v2, 13 November 2013)

\title{
Radiation hard silicon sensors for the CMS tracker upgrade
}

Thomas Poehlsen for the CMS Collaboration

\begin{abstract}
At an instantaneous luminosity of $5 \times 10^{34} \mathrm{~cm}^{-2} \mathrm{~s}^{-1}$, the high-luminosity phase of the Large Hadron Collider (HL-LHC) is expected to deliver a total of $3000 \mathrm{fb}^{-1}$ of collisions, hereby increasing the discovery potential of the LHC experiments significantly. However, the radiation dose of the tracking systems will be severe, requiring new radiation hard sensors for the CMS tracker. The CMS tracker collaboration has initiated a large material investigation and irradiation campaign to identify the silicon material and design that fulfils all requirements for detectors for the HL-LHC. Focussing on the upgrade of the outer tracker region, pad sensors as well as fully functional strip sensors have been implemented on silicon wafers with different material properties and thicknesses. The samples were irradiated with a mixture of neutrons and protons corresponding to fluences as expected for the positions of detector layers in the future tracker. Different proton energies were used for irradiations to investigate the energy dependence of the defect generation in oxygen rich material. The measurements performed on the structures include electrical sensor characterization, measurement of the collected charge injected with a beta source or laser light and bulk defect characterization. Measurements are performed at different annealing times. In this paper, results from the ongoing campaign are presented which led to the decision to take p-type silicon sensors for the CMS strip tracker.
\end{abstract}

Presented at IEEE-NSS-MIC-RTDS-2013 IEEE Nuclear Science Symposium and Medical Imaging Conference 


\title{
Radiation hard silicon sensors for the CMS tracker upgrade
}

\author{
Thomas Poehlsen on behalf of the CMS Tracker Collaboration
}

\begin{abstract}
At an instantaneous luminosity of $5 \times 10^{34} \mathrm{~cm}^{-2}$ $s^{-1}$, the high-luminosity phase of the Large Hadron Collider (HL-LHC) is expected to deliver a total of $3000 \mathrm{fb}^{-1}$ of collisions, hereby increasing the discovery potential of the LHC experiments significantly. However, the radiation dose of the tracking systems will be severe, requiring new radiation hard sensors for the CMS tracker. The CMS tracker collaboration has initiated a large material investigation and irradiation campaign to identify the silicon material and design that fulfils all requirements for detectors for the HL-LHC. Focussing on the upgrade of the outer tracker region, pad sensors as well as fully functional strip sensors have been implemented on silicon wafers with different material properties and thicknesses. The samples were irradiated with a mixture of neutrons and protons corresponding to fluences as expected for the positions of detector layers in the future tracker. Different proton energies were used for irradiations to investigate the energy dependence of the defect generation in oxygen rich material. The measurements performed on the structures include electrical sensor characterization, measurement of the collected charge injected with a beta source or laser light and bulk defect characterization. Measurements are performed at different annealing times. In this paper, results from the ongoing campaign are presented which led to the decision to take p-type silicon sensors for the CMS strip tracker.
\end{abstract}

\section{INTRODUCTION}

D URING the high-luminosity phase of the LHC (from around 2020 onwards)[1], the luminosity will be increased by a factor of about 5 with respect to the nominal LHC. Currently used oxygen-lean $320 \mu \mathrm{m}$ thick standard pon-n Float Zone (FZ) sensors will not withstand the resulting radiation damage which will be a $1 \mathrm{MeV}$ neutron equivalent fluence of $\Phi_{e q}=1.5 \times 10^{15} \mathrm{~cm}^{-2}$ after $3000 \mathrm{fb}^{-1}$ of collisions for the innermost layer of the strip detector at $r=20 \mathrm{~cm}$. Several new materials and production processes have been proposed and studied in recent years [2]. However, sensors produced for different experiments and the corresponding measurements are often hard to compare because they were done under different conditions, making strong conclusions difficult. CMS has therefore started a large campaign to systematically compare silicon materials, sensor designs, and layout parameters under otherwise identical conditions. The same small silicon sensors and test-structures for specific measurements were implemented by Hamamatsu Photonics K.K. on a variety of silicon wafers differing in bulk material, thickness, production process and polarity.

Results from the measurement campaign were presented at the last two IEEE conferences. Device characterization before irradiation was presented in [3], while neutron $(1 \mathrm{MeV})$ and

Thomas Poehlsen is with the Hamburg University, Germany. Email: thomas.poehlsen@desy.de proton $(23 \mathrm{MeV})$ irradiated samples were presented in [4]. This presentation will focus on the full-depletion voltages and on the seed signals for different annealing times after irradiation with a mixture of $23 \mathrm{GeV}$ protons and neutrons. The seed signal is the signal of the strip which has the highest signal of the whole sensor. This quantity is relevant for the binary readout chip which will be used for the highluminosity phase of CMS. For the detection of particles and the reconstruction of particle tracks only the seed signals will be relevant.

Oxygen-rich material is compared to material with mediumhigh oxygen concentrations, and both n-type and p-type sensors are studied.

\section{SENSORS}

This paper focusses on sensors for the outer layers of the tracker. For that, the materials and thicknesses investigated cover the relevant combination of parameters including the standard $320 \mu \mathrm{m}$ float zone material as a reference. $200 \mu \mathrm{m}$ thick standard FZ, Magnetic Czochralski (MCz) and moreover FZ produced with a new deep-diffusion (dd-FZ) process have been investigated.

Fully functional strip sensors and pad sensors were manufactured in both p-on-n (n-type) and n-on-p (p-type) technology. An overview over the materials relevant for this paper is given in Table I. The p-type sensors were produced with either p-stop strip isolation or with p-spray strip isolation.

\begin{tabular}{ccccc} 
material & active thickness $d$ & \multicolumn{2}{c}{$V_{\text {depl }}$} & oxygen \\
& & n-type & p-type & concentration \\
\hline FZ-200 & $200 \mu \mathrm{m}$ & $90 \mathrm{~V}$ & $120 \mathrm{~V}$ & $\sim 8 \times 10^{16} \mathrm{~cm}^{-3}$ \\
MCz-200 & $200 \mu \mathrm{m}$ & $150 \mathrm{~V}$ & $100 \mathrm{~V}$ & $\sim 5 \times 10^{17} \mathrm{~cm}^{-3}$ \\
dd-FZ-200 & $\sim 200 \mu \mathrm{m}$ & $100 \mathrm{~V}$ & $90 \mathrm{~V}$ & $\sim 3 \times 10^{17} \mathrm{~cm}^{-3}$ \\
dd-FZ-300 & $\sim 300 \mu \mathrm{m}$ & $190 \mathrm{~V}$ & $230 \mathrm{~V}$ & $\sim 1 \times 10^{17} \mathrm{~cm}^{-3}$ \\
\hline
\end{tabular}

FZ ... float-zone silicon

$\mathrm{MCz}$... magnetic Czochralski silicon

dd-FZ ...320 $\mu \mathrm{m}$ thick float-zone silicon wafers which underwent a deep diffusion process of dopants on the wafer backside to achieve different active thicknesses.

$V_{\text {depl }} \ldots$ full-depletion voltage. Voltage needed to fully deplete the sensor. TABLE I

OVERVIEW OVER THE MATERIALS USED.

\section{IRRADIATION}

All structures are electrically characterized before irradiation, followed by either neutron or proton irradiations to different fluences. After each irradiation, the structures are annealed and again electrically characterized. The structures initially exposed to neutron irradiation then receive a proton 
irradiation and vice versa, followed by another annealing treatment and electric characterization. This strategy allows to investigate the properties of the materials after pure proton and neutron irradiation and with mixed irradiations using a minimum number of material samples.

\section{RESUlts}

\section{A. Electrical sensor characterization using pad sensors}

The volume currents and their annealing behaviour has already been discussed for neutron irradiated and $23 \mathrm{MeV}$ irradiated samples of the same production in Ref. [4]. It has been found that the observed currents are compatible with the predictions by [5]. For the samples irradiated with 23 $\mathrm{GeV}$ protons (and neutrons) studied in this paper the same conclusion is true, and in the following paragraphs we will concentrate on the full-depletion voltages and on the seed signals.

The full-depletion voltage $V_{\text {depl }}$ for the FZ sensors after irradiation with $23 \mathrm{GeV}$ protons and after additional neutron irradiation is shown in Figure 1. For p-type sensors a continous increase of $V_{d e p l}$ is observed due to the dominating introduction of acceptors. In n-type sensors the introduction of acceptors leads to a decrease of $V_{d e p l}$ at low fluences and after type inversion to an increase of $V_{d e p l}$. Additional neutron irradiation leads to further generation of acceptors. This results in an increase of $V_{d e p l}$ in both p-type sensors and type inverted n-type sensors. At high fluences $V_{d e p l}$ is similar for p-type and n-type sensors, i.e. the radiation induced dopants dominate the effective doping concentration and the initially doping concentration is less relevant. However, slightly higher values of $V_{d e p l}$ in p-type sensors are observed as expected due to the remaining contributions of initial dopants.

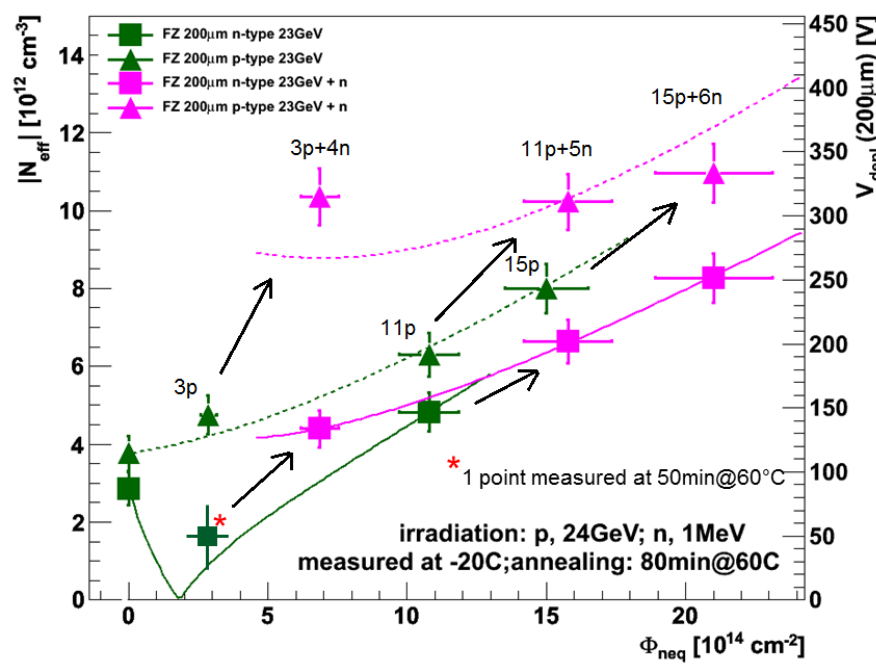

Fig. 1. Evolution of the effective doping concentration $\left(N_{e f f}\right)$ and the fulldepletion voltage $\left(V_{\text {depl }}\right)$ with the fluence for the FZ pad sensors irradiated with $23 \mathrm{GeV}$ protons and after additional neutron irradiation. Arrows and lines are to guide the eyes. The arrows indicate the change due to the additional neutron irradiation.

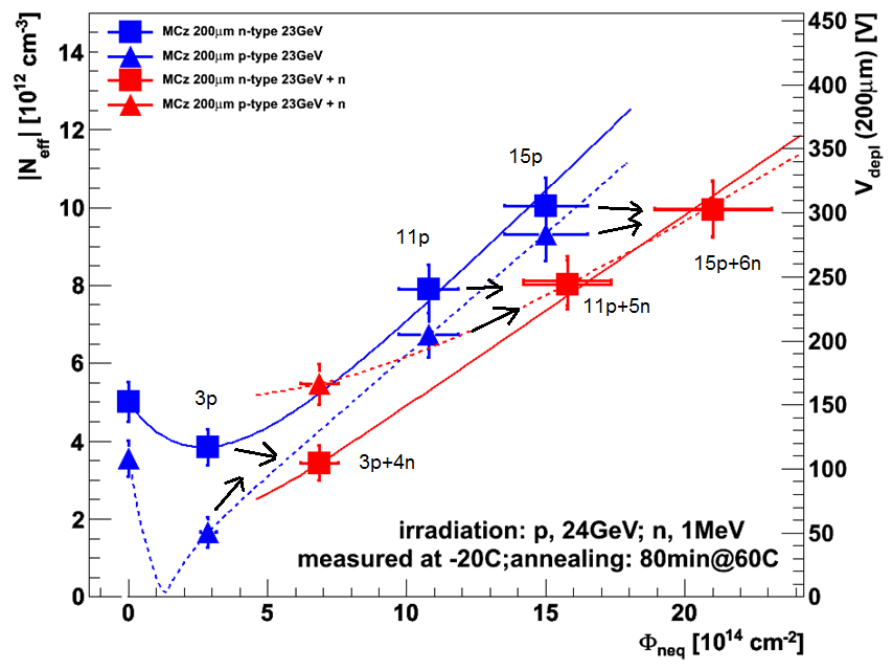

Fig. 2. Evolution of the effective doping concentration $\left(N_{e f f}\right)$ and the fulldepletion voltage $\left(V_{d e p l}\right)$ with the fluence for the $\mathrm{MCz}$ pad sensors irradiated with $23 \mathrm{GeV}$ protons and after additional neutron irradiation. Arrows and lines are to guide the eyes. The arrows indicate the change due to the additional neutron irradiation.

The full-depletion voltage $V_{d e p l}$ for the $\mathrm{MCz}$ sensors after irradiation with $23 \mathrm{GeV}$ protons and after additional neutron irradiation is shown in Figure 2. For n-type sensors at low proton fluences a small decrease in $V_{\text {depl }}$ is followed by a continous increase. The annealing behaviour of each point has also been studied and indicates that the sensors are still dominated by donors and that the n-type sensors did not undergo type inversion. This is compatible with expectations for high-oxygen material for which it is known that highenergy protons lead to an enhanced donor generation. The enhanced donor generation leads to type inversion in $\mathrm{MCz}$ type sensors. At higher proton fluences $V_{d e p l}$ is similar for p-type and n-type sensors, but this time slightly higher for ntype sensors. This is expected for type inverted p-type sensors due to the remaining contribution of the initial dopants. After additional neutron irradiation more acceptors are introduced to the donor-dominated sensors. Due to the compensation of donors and acceptors no strong increase in $V_{\text {depl }}$ is observed and in one case, at low fluences and for $\mathrm{MCz}$ n-type material, a decrease is observed. This compensation effect has already been observed in $\mathrm{MCz}$ n-type material [6]. Here it is observed that the effect is also beneficial in p-type $\mathrm{MCz}$ material (no increase of $V_{\text {depl }}$ ) compared to the $\mathrm{FZ}$ material with lower oxygen concentration (increase of $V_{d e p l}$ ).

The full-depletion voltage $V_{d e p l}$ as a function of annealing time is shown in Figure 3 for $\mathrm{FZ}$ and for $\mathrm{MCz}$ sensors after mixed irradiation. While the FZ sensors show a typical ptype annealing behaviour (decrease of $V_{\text {depl }}$ at low annealing times (beneficial annealing) and increase of $V_{\text {depl }}$ at higher annealing times (reverse annealing), the $\mathrm{MCz}$ sensors do not show this effect. Instead the full-depletion voltage continously decreases up to the measured annealing time of $400 \mathrm{~min}$ at $60{ }^{\circ} \mathrm{C}$ which is equivalent to about 7 weeks at $20{ }^{\circ} \mathrm{C}$. For 


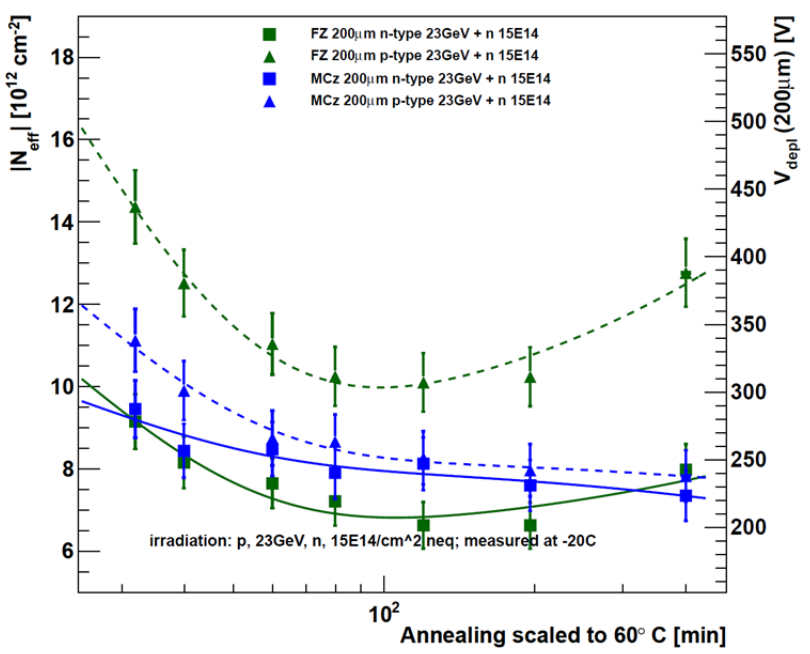

Fig. 3. Annealing behavior of the full-depletion voltage $\left(V_{d e p l}\right)$ for FZ and for $\mathrm{MCz}$ pad sensors irradiated with $\Phi_{e q}=1 \times 10^{15} \mathrm{~cm}^{-2}$ protons (23 $\mathrm{GeV})$ and $\Phi_{e q}=5 \times 10^{14} \mathrm{~cm}^{-2}$ neutrons. Lines are to guide the eyes.

a bias of $600 \mathrm{~V}$, which is the maximum bias voltage which can be achieved with the existing power supplies, no signal degradation is expected up to this annealing time, neither for FZ nor for $\mathrm{MCz}$, since $V_{d e p l} \ll 600 \mathrm{~V}$. However, at longer annealing times we expect signal degradation for FZ sensors due to reverse annealing.

\section{B. The seed signal in p-type strip sensors}

The seed signals for p-type sensors at $600 \mathrm{~V}$ bias are shown in Figure 4 as a function of annealing time. The FZ and $\mathrm{MCz}$ sensors are shown after irradiation with $23 \mathrm{GeV}$ protons and neutrons to the same fluences as the pad sensors shown in Figure 3. As expected from the pad sensor measurements for up to 7 weeks annealing at room temperature, no signal degradation is found in either $\mathrm{FZ}$ nor in $\mathrm{MCz}$ sensors. For longer annealing times, however, a signal degradation is found in FZ sensors due to reverse annealing. For up to 20 weeks at room temperature both $\mathrm{MCz}$ and $\mathrm{FZ}$ sensors provide seed signals of at least 8000 electrons. We conclude that p-type sensors of both $\mathrm{MCz}$ and $\mathrm{FZ}$ silicon are suited for an annealing scenario of 2 weeks at room temperature each year. $\mathrm{MCz}$ sensors are even suited for annealing sceanarios with longer annealing times. Longer annealing times would lead to a stronger reduction of the leakage current and hence to less power consumption and heat generation.

\section{Non-Gaussian noise in n-type strip sensors}

In n-type strip sensors significant non-Gaussian noise has been observed and leads to strip occupancies of more than $1 \%$ even if no particles are crossing the sensor. A typical noise distribution of an n-type strip sensor after mixed irradiation is shown in Figure 5. At low voltages the noise distribution is Gaussian while at high voltages significant noise tails may lead to noise hits, i.e. signals are present which exceed the threshold of $5 \sigma$.

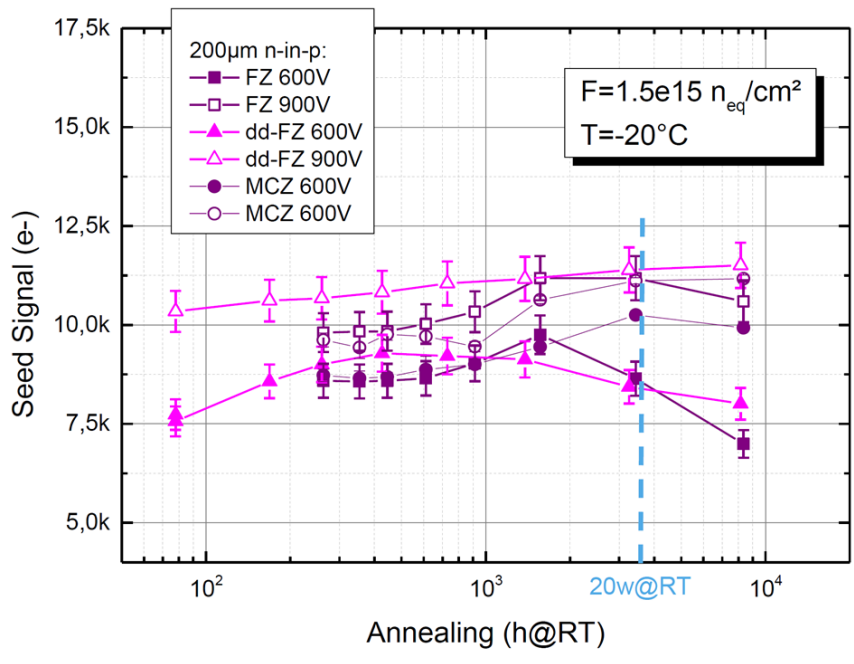

Fig. 4. The seed signal as a function of annealing time at room temperature after mixed irradiation. Sensors have been irradiated with $\Phi_{e q}=1 \times 10^{15} \mathrm{~cm}^{-2}$ protons and $\Phi_{e q}=5 \times 10^{14} \mathrm{~cm}^{-2}$ neutrons. Protons of $23 \mathrm{GeV}$ energy have been used for $\mathrm{FZ}$ and $\mathrm{MCz}$ sensors, protons of $23 \mathrm{MeV}$ have been used for dd-FZ sensors. Lines are to guide the eyes.

The strip occupancy due to non-Gaussian noise hits depends on bias voltage and annealing time, and it is less pronounced for neutron irradiation compared to irradiation with protons and neutrons. Figure 6 shows the strip occupancy due to noise hits as a function of bias voltage and annealing time. The strip occupancy is defined as the number of signals exceeding $5 \sigma$ devided by the number of strips and the number of events, i.e. a strip occupancy of $1 \%$ in a strip sensors with 100 strips corresponds to an average of one noise hit per event. While for mixed irradiation strip occupancies of above $1 \%$ are observed even at voltages of about $300 \mathrm{~V}$, for neutron irradiated sensors higher bias voltages of about $800 \mathrm{~V}$ are needed. This difference of charged and neutral hadrons indicates that ionising energy loss in the $\mathrm{SiO}_{2}$ interface might be relevant.

First simulations which take into account both the bulk defects generated by non-ionising energy loss and the increased oxide charge density due to ionising energy loss show very high electric fields close to the $\mathrm{p}+$ strip implants for n-type sensors. The electric field $0.1 \mu \mathrm{m}$ below the $\mathrm{SiO}_{2}$ is shown in Figure 7. An oxide charge density of $N_{o x}=1.2 \times 10^{12} \mathrm{~cm}^{-2}$ is assumed and bulk defects are implemented which correspond to a fluence of $\Phi_{e q}=1 \times 10^{15} \mathrm{~cm}^{-2}$. While in n-type sensors high electric fields are observed close to the p+ strip implants no field peaks are seen in p-type sensors. Lowering the values used for $N_{o x}$ also decreases the peak value of the electric field in n-type sensors.

\section{SUMMARY}

For the p-type sensors investigated in this work the seed signals are above 8000 electrons after mixed irradiation to a $1 \mathrm{MeV}$ neutron equivalent fluence of $\Phi_{e q}=1.5 \times 10^{15} \mathrm{~cm}^{-2}$, and up to an annealing time of 20 weeks at room temperature for both $\mathrm{MCz}$ and FZ sensors. $\Phi_{e q}=1.5 \times 10^{15} \mathrm{~cm}^{-2}$ is the expected fluence after $3000 \mathrm{fb}^{-1}$ of collisions for the innermost layer of the strip detector at $r=20 \mathrm{~cm}$. High 

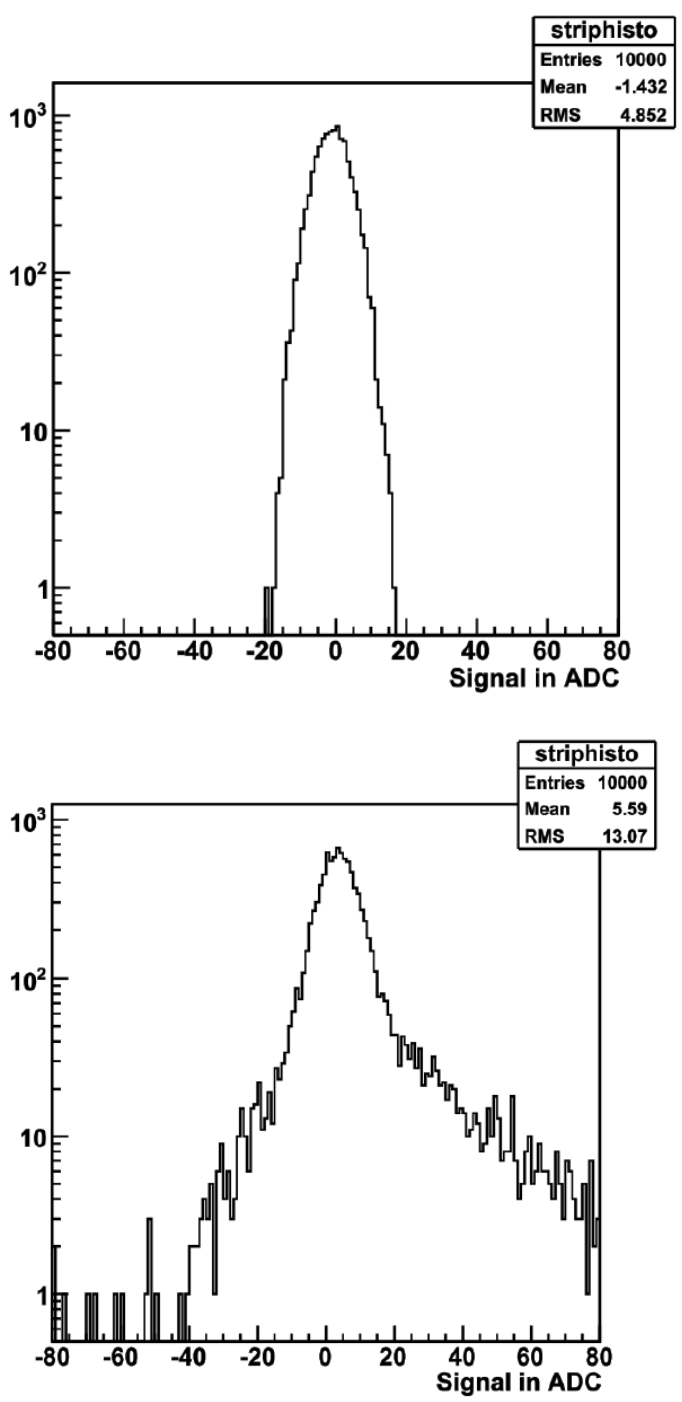

Fig. 5. Example of noise observed in irradiated n-type strip sensors taken from Ref. [7]. The pedestal-subtracted distribution is shown for a $200 \mu \mathrm{m}$ thick MCz n-type sensor after irradiation with $\Phi_{e q}=1 \times 10^{15} \mathrm{~cm}^{-2}$ protons $(23 \mathrm{MeV})$ and $\Phi_{e q}=5 \times 10^{14} \mathrm{~cm}^{-2}$ neutrons. Top: for $600 \mathrm{~V}$ bias. Bottom: for $900 \mathrm{~V}$ bias.

signals up to an annealing of 20 weeks at room temperature allow for an annealing sceanario with two weeks annealing for each winter shut down of the LHC. While for FZ sensors the signal degrades below 8000 electrons at 50 weeks at room temperature annealing time for $\mathrm{MCz}$ sensors no signal degradation is found. The operation of $\mathrm{MCz}$ sensors would enable annealing sceanarios with longer annealing times and hence with lower dark current, less power consumption and lower heat generation.

$\mathrm{MCz}$ sensors benefit from the compensation of neutrongenerated acceptors and proton-generated donors which is only present in the high-oxygen $\mathrm{MCz}$ material $\left(\sim 5 \times 10^{17}\right.$ $\mathrm{cm}^{-3}$ ) and not in the FZ material which has a lower oxygen concentration $\left(\sim 8 \times 10^{16} \mathrm{~cm}^{-3}\right)$. The compensation leads to low full-depletion voltages in $\mathrm{MCz}$ especially at long annealing times. The compensation effect is only observed for high-energy protons $(23 \mathrm{GeV})$ and neutrons, not for low-
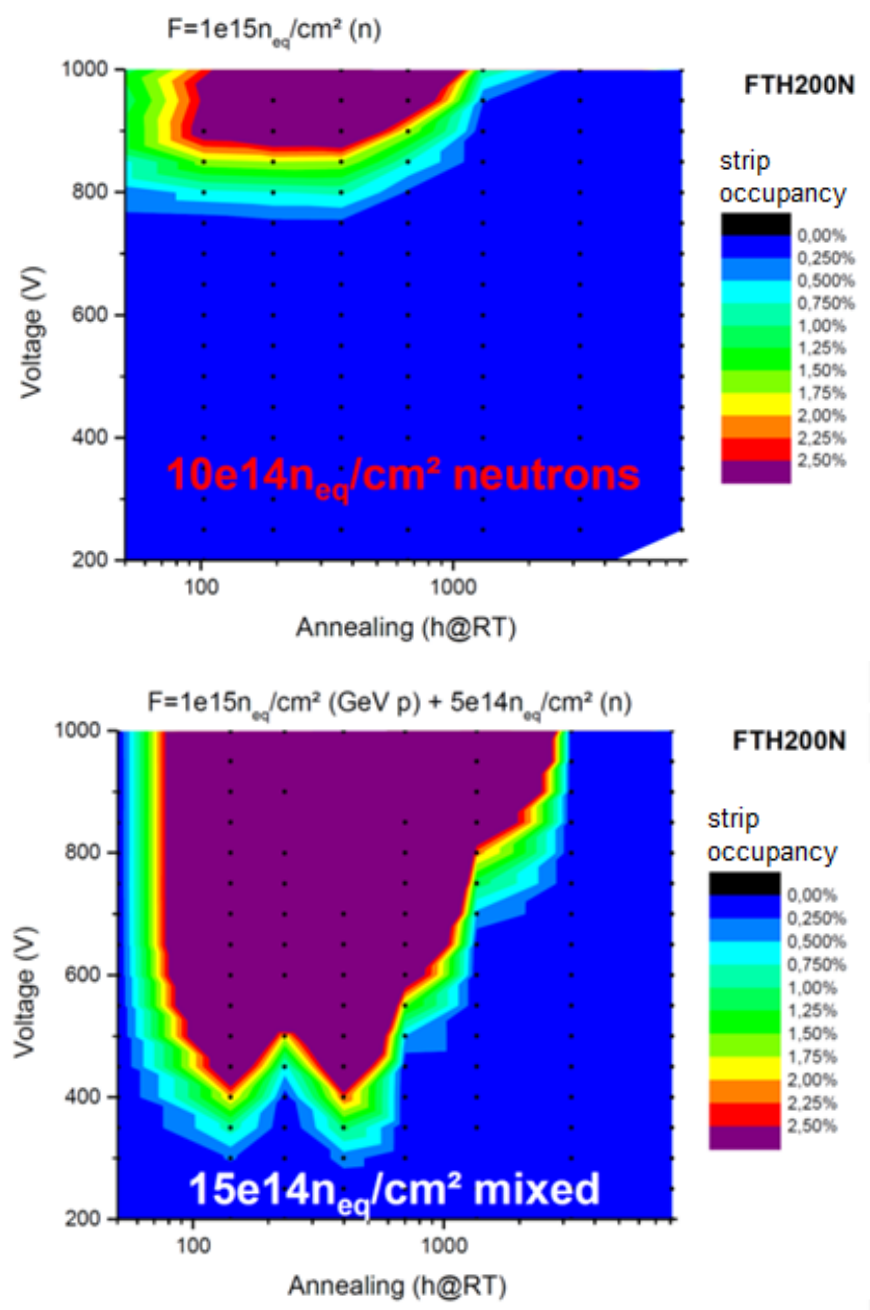

Fig. 6. Strip occupancy due to random noise hits as a function of bias voltage and annealing time after irradiation. The strip occupancy is defined as the number of signals exceeding $5 \sigma$ devided by the number of strips and the number of events. Top: $\Phi_{e q}=1 \times 10^{15} \mathrm{~cm}^{-2}$ neutrons. Bottom: $\Phi_{e q}=1 \times 10^{15} \mathrm{~cm}^{-2}$ protons $(23 \mathrm{GeV})$ and $\Phi_{e q}=5 \times 10^{14} \mathrm{~cm}^{-2}$ neutrons.

energy protons $(23 \mathrm{MeV})$ and neutrons. This can be understood since low-energy protons produce acceptors in both highoxygen and low-oxygen material [4], [8].

The n-type strip sensors investigated are affected by nonGaussian noise which leads to strip occupancies in the order of a few percent. The presence of non-Gaussian noise depends on the applied bias voltage and on the annealing state. First simulations indicate that the combination of bulk damage due to non-ionising energy loss and surface damage due to ionising energy loss lead to very high electric fields close to the p+ implants of n-type sensors.

Our measurements confirm that the bulk current is independent of the silicon material used, and conclusions from [4] and [5] stay valid. In order to limit the current generation of the sensors it is important to ensure cold operation temperatures of about $-20{ }^{\circ} \mathrm{C}$ at the sensor. Annealing during the winter shut downs can be used to further decrease the currents, up to two weeks at room temperature if FZ sensors are used, or 


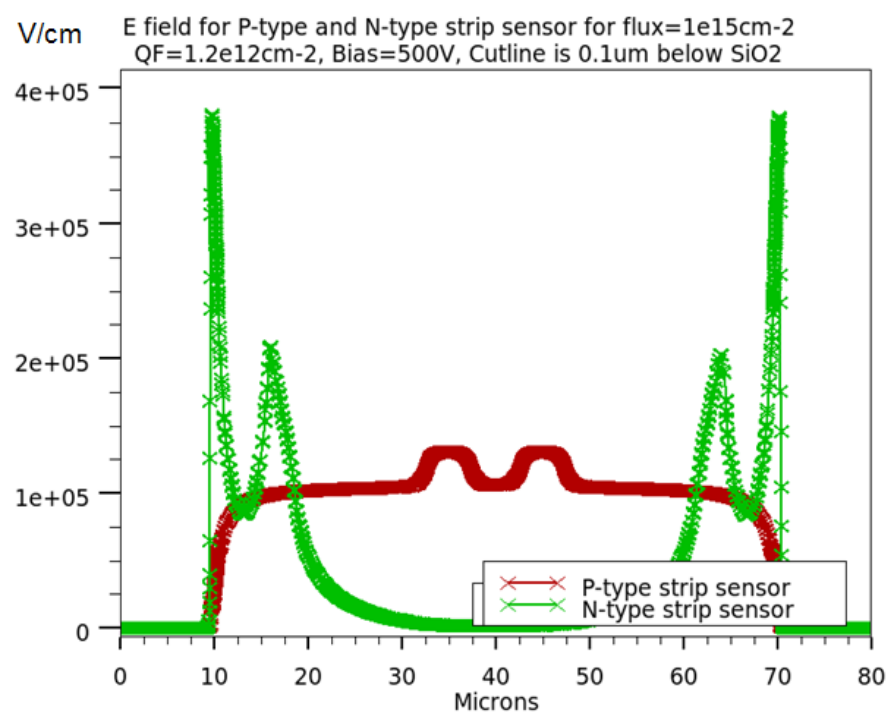

Fig. 7. Simulated absolute value of the electric field at $0.1 \mu \mathrm{m}$ below the $\mathrm{SiO}_{2}$. The simulation is performed for a bias voltage of $500 \mathrm{~V}$, an oxide charge density of $N_{o x}=1.2 \times 10^{12} \mathrm{~cm}^{-2}$ to account for surface radiation damage due to ionising energy loss, and bulk defects which are tuned to describe the electric field in the sensor bulk after an irradiation damage due to non-ionising energy loss of $\Phi_{e q}=1 \times 10^{15} \mathrm{~cm}^{-2}$. The gap center is at $x=40 \mu \mathrm{m}$, the strip implants are $20 \mu \mathrm{m}$ wide (from 0 to 10 and from 70 to 80), the aluminium overhang is about $6 \mu \mathrm{m}$.

even longer annealing times if $\mathrm{MCz}$ sensors are used.

Due to the good performance of the p-type sensors after the expected fluence for the innermost strip layer, and due to the significant number of non-Gaussian noise hits that has been observed in n-type sensors, CMS decided to use p-type silicon sensors for the high-luminosity-phase strip tracker.

\section{REFERENCES}

[1] E. Shaposhnikova, CERN-AB-2008-065 (2008).

[2] The RD50 Collaboration, CMS-CR-2011-308 (2011).

[3] M. Bergholz for the CMS Tracker Collaboration, CERN-LHCC-2010012 (2010).

[4] G. Steinbrück for the CMS Tracker Collaboration, CMS CR-2012/308 (2012).

[5] Michael Moll, Radiation Damage in Silicon Particle Detectors Microscopic Defects and Macroscopic Properties, PhD thesis, Hamburg University, 1999.

[6] G. Kramberger et al., Nucl. Instr. and Meth. A 609 (2009) 142-148.

[7] A. Dierlamm on behalf of the CMS Tracker Collaboration, PoS(Vertex 2012)016.

[8] N. Manna et al., Nucl. Instr. and Meth. A 583 (2007) 87-90. 